\title{
CARACTERIZACIÓN DE LAS PROPIEDADES FÍSICO MECÁNICAS DE ESPECIES, PINO (Pinus patula) Y TORNILLO (Cedrelinga cateniformis) PROVENIENTES DEL PERÚ UTILIZANDO TECNICAS NO DESTRUCTIVAS
}

\author{
CHARACTERIZATION OF THE PHYSICAL MECHANICS PROPERTIES OF THE \\ PERUVIAN SPECIES, PINO (Pinus patula) AND TORNILLO (Cedrelinga cateniformis) BY \\ USING NON DESTRUCTIVES TECHNIQUES
}

\author{
${ }^{1}$ Yoza L., ${ }^{2}$ Baradit E. \& ${ }^{3}$ Acevedo M.
}

\begin{abstract}
Resumen
El objetivo del presente trabajo, fue la determinación de las propiedades físico mecánicas de 2 especies de maderas de bosques peruanos el pino patula y el tornillo, utilizando métodos no destructivos de ultrasonido, especies maderables usadas en estructuras de madera. En el cálculo de estructuras a nivel de ingeniería, comúnmente se usa la información de las propiedades básicas como el módulo de elasticidad de flexión, módulo de compresión paralela y módulo de cizallamiento longitudinal. En los últimos años gracias al avance alcanzado con los métodos no destructivos de ultrasonido se han podido determinar con mas facilidad los módulos de elasticidad en las otras direcciones como, $\mathrm{E}(\mathrm{RR})$; $\mathrm{E}(\mathrm{TT})$, y de cizallamiento $\mathrm{G}(\mathrm{RL}), \mathrm{G}(\mathrm{TR})$ permitiendo, con esta información, mejorar el diseño de estructuras y la reducción de costos en materiales. En el presente trabajo, usando el equipo de ultrasonido Parametric PR 5800 y transductores con frecuencia de $1 \mathrm{MHz}$ se caracterizaron las 2 especies mencionadas. Para lo cual, primeramente se midieron las velocidades de propagación de las ondas ultrasónicas tanto longitudinales como transversales en las direcciones principales y luego se calcularon los módulos elásticos respectivos obteniéndose valores comparables con los obtenidos en las pruebas mecánicas tradicionales, siendo esta la primera vez que se obtienen en el Perú con este método.
\end{abstract}

Palabras claves: Maderas peruanas, ultrasonido, módulos de elasticidad.

\begin{abstract}
The objective of this study was the determination of the physical mechanics properties of 2 species of Peruvian forest, Pino patula and Tornillo using non-destructive methods, species used very common in the design of wood structures.

The structures design at the level of engineering commonly uses the information on the basic properties as the transverse elasticity module, module compression and shearing module. In recent years thanks to the advances achieved with ultrasound acoustic non-destructive methods have been able to determine the modulus of elasticity in other directions such as, E (RR); (E) (TT), G (RL), G (TR) allowing improving the design of structures managing the reduction of costs in materials.In this paper using a Parametric PR 5800 Ultrasound $1 \mathrm{MHz}$ frequency equipment, to characterize these 2 species which were first measured velocities of propagation of longitudinal and transverse acoustic waves in the main directions and then calculated the elastic respective elastic modules obtaining values comparable with those obtained in traditional mechanical tests. Point out, that is first time that we get in Peru with this method.
\end{abstract}

Key words: Peruvian Woods, ultrasound, elastic modulus.

\section{Introducción}

El conocimiento de las propiedades físico-mecánicas de la madera juega un rol importante en el momento de determinar su uso tanto en la industria de la construcción como en la de muebles. En este sentido se hace cada vez más necesario mejorar el conocimiento de las propiedades físico-mecánicas de las especies de nuestros bosques por un lado para optimizar el uso del recurso y por otro lado para hacer más competitiva la industria maderera nacional. Para lo cual, haremos uso de las técnicas acústicas no destructivas y pruebas mecánicas tradicionales para caracterizar las especies pino patula y tornillo. En este sentido, el pino (Pinus patula) es una especie que viene introduciéndose en el mercado por lo cual presenta un interés creciente para su explotación. La otra madera es tornillo (Cedrelinga cateniformis) especie muy utilizada en el diseño de estructuras la cual servirá

\footnotetext{
${ }^{1}$ Universidad Nacional Agraria La Molina,Lima, Perú. E-mail: lyoza@lamolina.edu.pe ${ }^{2}$ Departamento de Física, Facultad de Ciencias, Universidad del Bío Bío, Concepción, Chile.

${ }^{3}$ Universidad Nacional Agraria La Molina, Lima, Perú.
} 
como referencia comparativa en el presente estudio, y valorar el uso del pino como madera estructural.

\section{Pino (Pinus patula)}

Esta especie crece en las formaciones vegetales como el bosque húmedo o muy húmedo montano bajo. En su distribución natural forma parte de los bosques nublados y asociado con las especies: Pinus ayacahuite, Pinus michoacana, Pinus lumholtzii y Pinus leiophylla. En Colombia y otros países donde se ha introducido la especie, ha presentado un excelente desarrollo, convirtiéndose en una especie maderable y útil para programas de reforestación en zonas altas. En el Perú se encuentran en el Departamento de Cajamarca. Es un árbol de 10 a $25 \mathrm{~m}$ de altura total, de corteza escamosa y roja, sobre todo en la parte superior del tronco, el tallo es generalmente recto, con trozas comerciales entre 10 y $20 \mathrm{~m}$ de longitud y diámetros entre 25 a $50 \mathrm{~cm}$. Las características de la madera son:

En condición seca al aire la albura es de color blanco amarillento y el duramen pardo amarillento a pardo marrón. Anillos de crecimiento no diferenciados. Grano recto, textura fina y veteado en arcos superpuestos y bandas paralelas.

A nivel macro y microscópico el parénquima es ausente, traqueidas longitudinales: visibles con lupa 10x. Son células largas y anchas, con presencia de puntuaciones areoladas en su interior. Sin marcada diferencia entre traqueidas de primavera y traqueidas de verano. La longitud varía de 1150 a $4500 \mu \mathrm{m}$ y su ancho de 30 a $60 \mu \mathrm{m}$. Las punteaduras en el campo de cruzamiento son del tipo pinoide. Los radios son uniseriados y fusiformes; estos últimos con presencia de canal resinífero cuya altura es de una a catorce células y de 141 a $471 \mu \mathrm{m}$. Presencia de canales resiníferos de dos tipos, longitudinales esparcidos entre las traqueidas longitudinales y radiales, incluidos dentro de los radios fusiformes. La figura1 ilustra los cortes en secciones transversal y tangencial.
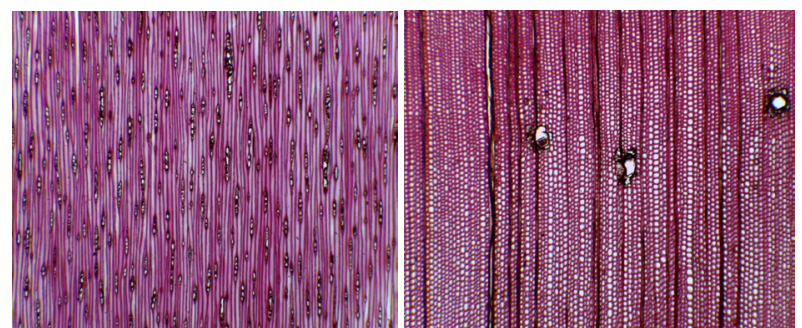

Figura 1. Vistas microscópicas de $(40 \mathrm{x})$ en corte transversal y longitudinal del pino.

Fuente: Laboratorio de Anatomía de la Madera UNALM

\section{Tornillo (Cedrelinga cateniformis)}

Esta especie se distribuye en la región amazónica de Bolivia, Brasil, Colombia, Ecuador y Perú en altitudes de hasta 700msnm. En el Perú se encuentran en los
Departamentos de Cuzco, Huánuco, Loreto, Madre de Dios, San Martín y Ucayali

Es un árbol de 20 a $40 \mathrm{~m}$ de altura total y de 15 a $30 \mathrm{~m}$ de altura comercial, fuste cilíndrico y diámetro de 0,15 a $1,2 \mathrm{~m}$. Su nombre científico es Cedrelinga cateniformis, cuyas características son:

En condición seca al aire la albura es de color rosado claro y el duramen rosado oscuro. Anillos de crecimiento no diferenciados. Grano recto a entrecruzado, textura gruesa y veteado ausente.

A nivel macro y microscópica los poros son visibles a simple vista, solitarios de forma ovalada. Porosidad difusa, mayormente solitarios de forma ovalada y escaso múltiple radiales de 2 a 4 poros. Diámetro tangencial promedio de 280 a $320 \mu \mathrm{m}$; con 1 a 5 poros $/ \mathrm{mm}^{2}$. La longitud promedio de los elementos vasculares va de 405 a $445 \mu \mathrm{m}$, platina de perforación simple, punteaduras intervasculares alternas de forma redonda y apertura incluida. Parénquima visible con lupa 10x, paratraqueal vasicéntrico; con presencia de parénquima apotraqueal difuso, en corte longitudinal no estratificado. Presencia de inclusiones gomosas. Los radios visibles con lupa 10x, finos y pocos contrastados en sección radial. En sección tangencial uniseriados, con altura entre 240 y $345 \mu \mathrm{m}$. En sección radial homocelulares formado por células procumbentes. Con 5 a 10 radios $/ \mathrm{mm}$; no estratificados. Punteaduras radiovasculares similares a las intervasculares. Presencia de inclusiones gomosas. Las fibras son libriformes con puntuaciones simples, no estratificadas; con longitud entre 1300 y $1450 \mu \mathrm{m}$, diámetro total de $15 \mu \mathrm{m}$ y espesor de pared celular de $1,4 \mu \mathrm{m}$. En la Figura 2 se ilustra los cortes transversal y tangencial.

Esta madera se usada en crucetas, puntales, encofrados, tijerales, vigas, viguetas, puertas, ventanas, tabiquería, machihembrado, mobiliario en general, parihuelas y carrocerías.
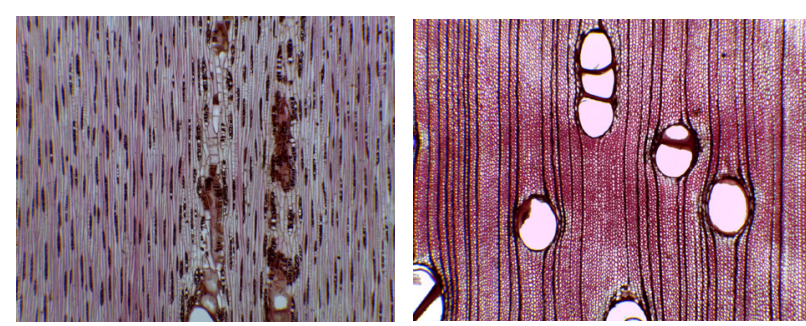

Figura 2. Vistas microscópicas (40x) en corte transversal y tangencial del tornillo.

Fuente: Laboratorio de Anatomía de la Madera UNALM

\section{Evaluación no destructiva.}

Comúnmente, la información de las propiedades físicomecánicas proviene como resultado de la realización de diferentes mediciones tradicionales en los que una máquina de ensayos juega el papel preponderante. Sin embargo, en los últimos años se han implementado diferentes técnicas no destructivas tanto para la evaluación de los materiales, como para diferentes procesos de clasificación. Entre 
estas técnicas una de las más utilizadas es la basada en la propagación de ondas mecánicas de diferente frecuencia, fundamentalmente en la banda ultrasónica. Cuya ventaja es usarlas por ejemplo, para determinar las constantes elásticas de diferentes materiales tales como en maderas (Baradit 2012, Kranitz 2012) y también en hormigón y morteros (Rodríguez 2003, Lawson et al. 2011).

En particular los módulos de elasticidad de Young (E) se obtienen para las tres direcciones principales del árbol por medio de la siguiente ecuación.

$$
E_{\ddot{i}}=\rho v^{2}
$$

Donde $\mathrm{i}=1,2,3$ indica cada uno de los ejes principales y $\mathrm{V}$ es la velocidad de propagación longitudinal del pulso ultrasónico (Bucur 2006). Los módulos de cizallamiento $\mathrm{G}$ se obtienen por medio de una expresión similar.

$$
G_{i j}=\rho v^{2}
$$

Donde $i \neq j$, V es la velocidad de propagación del pulso con polarización transversal.

\section{Materiales y métodos}

\section{Muestras.}

Las muestras fueron seleccionadas de 5 árboles por especie de pino y tornillo. Las probetas para las pruebas mecánicas fueron preparadas de acuerdo con la norma ASTM D143 (2008). Las pruebas de flexión estática se efectuaron con un método secundario con las dimensiones de las muestras $25 \times 25 \times 410 \mathrm{~mm}$ con un total de 6 muestras para el pino y 8 para el tornillo. Las pruebas de compresión paralela se hicieron muestras de dimensiones $50 \times 50 \times 200 \mathrm{~mm}$, con un total de 6 muestras para el pino y 8 para el tornillo. Para las pruebas de cizallamiento se usaron 9 muestras para el pino y 16 para el tornillo respectivamente.

Las muestras para las pruebas de ultrasonido fueron tomadas conjuntamente con las de las pruebas mecánicas

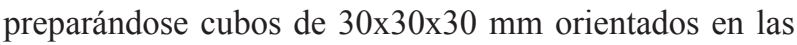

direcciones L.R.T de los 5 árboles escogidos por especie obteniéndose 19 muestras para la especie Pino y 18 para el tornillo.

\section{Equipos}

El montaje experimental se muestra en el diagrama de bloque de la figura 3 y consiste en un generador de ultrasonido marca Panametric PR 5800 y un osciloscopio marca Tektronic. Los transductores utilizados fueron de 1 $\mathrm{MHz}$ de frecuencia tanto para la generación de ondas de polarización longitudinal como transversal.

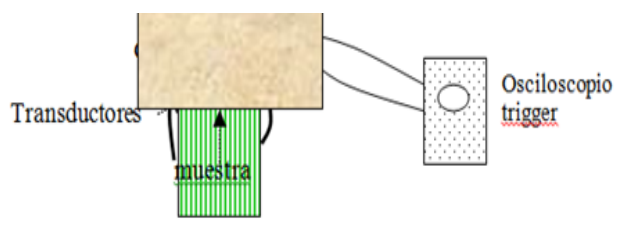

Figura 3. Disposición del equipamiento de medición.

\section{Resultados y Discusión}

Losresultados de las velocidades en las distintas direcciones son mostrados en las tablas 1 y 2. El comportamiento de las velocidades es muy similar en ambas especies cumpliéndose la relación típica $\mathrm{V}(\mathrm{LL})>\mathrm{V}(\mathrm{RR})>\mathrm{V}(\mathrm{TT})$. Otras típicas relaciones están vinculadas con la simetría de las velocidades de corte $\mathrm{V}(\mathrm{LR})=\mathrm{V}(\mathrm{RL}), \mathrm{V}(\mathrm{LT})=\mathrm{V}(\mathrm{TL})$ y $\mathrm{V}(\mathrm{RT})=\mathrm{V}(\mathrm{TR})$. Estas igualdades como resultado de las mediciones experimentales son obviamente aproximadas (tabla 1 y 2).

Podemos señalar para el caso del tornillo que los coeficientes de variación para las velocidades $\mathrm{V}_{\mathrm{TT}}, \mathrm{V}_{\mathrm{TL}} \mathrm{y}$ $\mathrm{V}_{\mathrm{LT}}$ son significativamente más altos comparados con el pino, posiblemente porque fueron extraídas de bosque naturales.

Tabla 1. Velocidad de la onda longitudinal y transversal para el Pinus patula.

\begin{tabular}{cccccccccc}
\hline $\begin{array}{c}\text { Velocidad } \\
(\mathrm{m} / \mathrm{s})\end{array}$ & $\mathrm{V}_{\mathrm{LL}}$ & $\mathrm{V}_{\mathrm{RR}}$ & $\mathrm{V}_{\mathrm{TT}}$ & $\mathrm{V}_{\mathrm{RL}}$ & $\mathrm{V}_{\mathrm{TL}}$ & $\mathrm{V}_{\mathrm{TR}}$ & $\mathrm{V}_{\mathrm{LR}}$ & $\mathrm{V}_{\mathrm{LT}}$ & $\mathrm{V}_{\mathrm{RT}}$ \\
\hline media & 4578 & 2022 & 1257 & 1523 & 1335 & 541 & 1603 & 1341 & 537 \\
DS. & 231 & 120 & 27 & 82 & 113 & 47 & 125 & 86 & 35 \\
CV.(\%) & 5,0 & 5,9 & 2,2 & 5,4 & 8,4 & 8,7 & 7,8 & 6,4 & 6,5 \\
\hline
\end{tabular}

Tabla 2. Velocidad de la onda longitudinal y transversal para el Cedrelinga cateniformis.

\begin{tabular}{cccccccccc}
\hline $\begin{array}{c}\text { Velocidad } \\
(\mathrm{m} / \mathrm{s})\end{array}$ & $\mathrm{V}_{\mathrm{LL}}$ & $\mathrm{V}_{\mathrm{RR}}$ & $\mathrm{V}_{\mathrm{TT}}$ & $\mathrm{V}_{\mathrm{RL}}$ & $\mathrm{V}_{\mathrm{TL}}$ & $\mathrm{V}_{\mathrm{TR}}$ & $\mathrm{V}_{\mathrm{LR}}$ & $\mathrm{V}_{\mathrm{LT}}$ & $\mathrm{V}_{\mathrm{RT}}$ \\
\hline media & 4495 & 1860 & 1231 & 1411 & 1204 & 676 & 1330 & 1205 & 696 \\
DS. & 247 & 58 & 156 & 75 & 160 & 53,0 & 71 & 137 & 40 \\
CV.(\%) & 5,5 & 3,1 & 12,7 & 5,3 & 13,2 & 7,8 & 5,4 & 11,3 & 5,7 \\
\hline
\end{tabular}


Los resultados de las velocidades de polarización longitudinal muestran una anisotropía elástica en relación a los tres ejes principales similar es para ambas especies. En el caso del pino 1:2,3:3,6 mientras que para el tornillo 1:2,4:3,6. Dichos valores de anisotropía son levemente más altos que para maderas coníferas (softwoods) chilenas pero más bajos que para latifoliadas (hardwoods), (Baradit 2012).
En las tablas 3 y 4 se presentan los valores de los módulos elásticos tanto para el pino y tornillo. Los módulos de elasticidad de Young se muestran muy similares para ambas especies y sólo en la dirección radial se observa una pequeña diferencia, mientras tanto, para los módulos de corte la diferencia entre ambas especies es más significativa.

Tabla 3. Modulo de elasticidad y cizallamiento para el Pinus patula.

\begin{tabular}{lccccccc}
\hline & $\begin{array}{c}\text { Densidad } \\
\left(\mathrm{kg} / \mathrm{m}^{3}\right)\end{array}$ & $\begin{array}{c}\mathrm{E}_{\mathrm{LL}} \\
(\mathrm{MPa})\end{array}$ & $\begin{array}{c}\mathrm{E}_{\mathrm{RR}} \\
(\mathrm{MPa})\end{array}$ & $\begin{array}{c}\mathrm{E}_{\mathrm{TT}} \\
(\mathrm{MPa})\end{array}$ & $\begin{array}{c}\mathrm{G}_{\mathrm{LR}} \\
(\mathrm{MPa})\end{array}$ & $\begin{array}{c}\mathrm{G}_{\mathrm{TL}} \\
(\mathrm{MPa})\end{array}$ & $\begin{array}{c}\mathrm{G}_{\mathrm{TR}} \\
(\mathrm{MPa})\end{array}$ \\
\hline media & 508 & 10780 & 2077 & 805 & 1240 & 908 & 153 \\
DS. & 55,4 & 2087,2 & 98,8 & 10,5 & 139,9 & 85,0 & 34,6 \\
CV. & 10,9 & 19,4 & 4,8 & 1,3 & 11,3 & 9,4 & 22,6 \\
\hline
\end{tabular}

Tabla 4. Modulo de elasticidad y cizallamiento para el Cedrelinga cateniformis.

\begin{tabular}{lccccccc}
\hline & $\begin{array}{c}\text { Densidad } \\
\left(\mathrm{Kg} / \mathrm{m}^{3}\right)\end{array}$ & $\begin{array}{c}\mathrm{E}_{\mathrm{LL}} \\
(\mathrm{MPa})\end{array}$ & $\begin{array}{c}\mathrm{E}_{\mathrm{RR}} \\
(\mathrm{MPa})\end{array}$ & $\begin{array}{c}\mathrm{E}_{\mathrm{TT}} \\
(\mathrm{MPa})\end{array}$ & $\begin{array}{c}\mathrm{G}_{\mathrm{LR}} \\
(\mathrm{MPa})\end{array}$ & $\begin{array}{c}\mathrm{G}_{\mathrm{LT}} \\
(\mathrm{MPa})\end{array}$ & $\begin{array}{c}\mathrm{G}_{\mathrm{RT}} \\
(\mathrm{MPa})\end{array}$ \\
\hline media & 528 & 10702 & 1831 & 814 & 997 & 777 & 251 \\
DS. & 28,9 & 1321,2 & 188,3 & 216,6 & 118,3 & 150,2 & 39,2 \\
CV. & 5,3 & 12,3 & 10,3 & 26,6 & 11,9 & 19,3 & 15,6 \\
\hline
\end{tabular}

En la tablas 5 y 6 se muestran los valores de las propiedades mecánicas para ambas especies.

Señalándose los resultados de las pruebas en flexión estática: pl, esfuerzo al límite proporcional, (proportional limite stress); MOR, módulo de ruptura (modulus of rupture); MOE, módulo de elasticidad (modulus of elasticity) y las de compresión paralela al grano: $\mathrm{pl}_{\mathrm{L}}$, esfuerzo limite proporcional (proportional limite stress longitudinal); $\mathrm{MOR}_{\mathrm{L}}$ módulo de ruptura ( modulus of rupture longitudinal); $\mathrm{MOE}_{\mathrm{L}}$ módulo de elasticidad (modulus of elasticity longitudinal) y además del cizallamiento paralelo al grano, $\operatorname{SHEAR}_{\mathrm{L}}$ (shear strenght, longitudinal).

Tabla 5. Propiedades mecánicas del Pinus patula.

\begin{tabular}{cccccccc}
\hline & \multicolumn{2}{c}{$\begin{array}{c}\text { Flexión Estática, } M(12 \%) \\
(\mathrm{MPa})\end{array}$} & \multicolumn{2}{c}{$\begin{array}{c}\text { Compresión, } M(16 \%) \\
(\mathrm{MPa})\end{array}$} & $\begin{array}{c}\text { Cizallamiento, } M(14 \%) \\
(\mathrm{MPa})\end{array}$ \\
\hline & $\mathrm{Pl}$ & $\mathrm{MOR}$ & $\mathrm{MOE}$ & $\mathrm{pl}_{\mathrm{L}}$ & $\mathrm{MOR}_{\mathrm{L}}$ & $\mathrm{MOE}_{\mathrm{L}}$ & Cizallamiento $_{\mathrm{L}}$ \\
media & 72 & 110 & 8012 & 19 & 25 & 8453 & 10 \\
SD. & 25,5 & 33,7 & 1999,2 & 2,7 & 3,2 & 1858,6 & 2,3 \\
CV.(\%) & 35,6 & 30,8 & 24,9 & 14,2 & 12,7 & 22,0 & 22,8 \\
\hline
\end{tabular}

Tabla 6. Propiedades mecánicas del Cedrelinga cateniformi.

\begin{tabular}{|c|c|c|c|c|c|c|c|}
\hline & \multicolumn{3}{|c|}{$\begin{array}{l}\text { Flexión Estática, } M(12 \%) \\
(\mathrm{MPa})\end{array}$} & \multicolumn{3}{|c|}{$\begin{array}{c}\text { Compresión, } M(17 \%) \\
(\mathrm{MPa})\end{array}$} & \multirow{2}{*}{$\begin{array}{c}\begin{array}{c}\text { Cizallamiento, } M(12 \%) \\
(\mathrm{MPa})\end{array} \\
\text { Cizallamiento }_{\mathrm{L}}\end{array}$} \\
\hline & $\mathrm{pl}$ & MOR & MOE & $\mathrm{pl}_{\mathrm{L}}$ & $\mathrm{MOR}_{\mathrm{L}}$ & $\mathrm{MOE}_{\mathrm{L}}$ & \\
\hline media & 35 & 58 & 8268 & 22 & 30 & 10404 & 10 \\
\hline SD. & 6,2 & 9,45 & 1198,3 & 1,9 & 2,5 & 1455,5 & 1,2 \\
\hline CV.(\%) & 17,5 & 16,2 & 14,5 & 8,7 & 8,2 & 14,0 & 11,0 \\
\hline
\end{tabular}

Se observa que para los ensayos de flexión los módulos de elasticidad son similares para ambas especies, siendo para el tornillo más alto en solo unos $200 \mathrm{MPa}$, mientras que el módulo de ruptura para el tornillo es casi la mitad que para el pino. En compresión paralela, el módulo de elasticidad para el tornillo resultó unos $2000 \mathrm{MPa}$ mayor que para el pino, mientras que los módulos de ruptura son similares en ambas especies.

\section{Conclusiones}

El comportamiento de las velocidades es muy similar en ambas especies cumpliéndose la relación típica $\mathrm{V}(\mathrm{LL})>\mathrm{V}(\mathrm{RR})>\mathrm{V}(\mathrm{TT})$ y cumpliendo las relaciones de simetría de las velocidades de corte $\mathrm{V}(\mathrm{LR})=\mathrm{V}(\mathrm{RL})$, $\mathrm{V}(\mathrm{LT})=\mathrm{V}(\mathrm{TL})$ y $\mathrm{V}(\mathrm{RT})=\mathrm{V}(\mathrm{TR})$. Podemos señalar que para tornillo que los coeficientes de variación para las 
velocidades $\mathrm{V}_{\mathrm{TT}}, \mathrm{V}_{\mathrm{TL}}$ y $\mathrm{V}_{\mathrm{LT}}$ son significativamente más altos comparados con el pino, posiblemente porque fueron extraídas de bosque naturales. Mostrando velocidades polarizadas longitudinalmente anisotropías elástica de 1:2,3:3,6 y 1:2,4:3,6. para el pino y tornillo respectivamente. Los módulos de elasticidad de Young se muestran muy similares para ambas especies, mientras tanto, para los módulos de corte la diferencia es más significativa.

En ensayos mecánicos de flexión los módulos de elasticidad son similares para ambas especies, siendo para el tornillo mayor en $200 \mathrm{MPa}$, mientras que el módulo de ruptura para el tornillo es casi la mitad que para el pino. En compresión paralela, el módulo de elasticidad para el tornillo resultó $2000 \mathrm{MPa}$ mayor, mientras que los módulos de ruptura son similares en ambas especies.

En estudios futuros se recomienda la medición de los coeficientes de Poisson así como la evaluación de maderas de tornillo perteneciente a plantación

\section{Literatura citada}

ASTM (American Standard Test Methods) D143. Standard Test Methods. 2008. for small clear specimens of timber.

Baradit, E. and Niemz, P. 2012. Some Elastic Constants of the Chilean Wood Species: tepa, olivillo, laurel, lenga, alerce and manio using ultrasound techniques. Wood Research 57(3):497-504.

Baradit, E.; Keunecke, D. and Niemz, P. 2012. Stifness Moduli of various extraneous species determined with ultrasound. Wood Research 57(1):173-178.

Bucur, V. 2006. Acoustic of Wood, Springer. Berlin.

Kranitz, K.; Baradit E.; Dobrowolska E.; Plotze M. and Niemz, P. 2012. Untersuchungen zu Eingenschaften von Mooreiche. Holztechnologie 53(1):11-17.

Lawson, I.; Danso H. C.; Odoi, H. C.; Adjei, C. A.; Quashie, I. I.;Mumuni, I. I. and Ibrahim, I. S. 2011. Non-Destructive evaluation of concrete using ultrasound pulse velocity. Research Journal of Applied Sciences, Engineering and Technology 3(6):499-504.

Rodríguez, G. S. y Pazini, E. 2003. Módulo de elasticidad estático del hormigón determinado por medio de pruebas de ultrasonido. Materiales de Construcción, 53 (271-272): 47-55.

Rosell, J. R. y Cantalapiedra, I. R. 2011. Método simple para determinar el módulo de Young dinámico a partir de una excitación por impacto aplicado a morteros de cal y cemento. Materiales de Construcción, 61 (301): 39-48. 